\title{
Influencia del clima motivacional en educación física sobre las metas de logroy la satisfacción con la vida de los adolescentes \\ Influence of motivational climate in physical education on achievement goals and adolescents' life satisfaction \\ Melchor Gutiérrez, Jose M. Tomás, Pablo Calatayud \\ Universidad de Valencia(España)
}

\begin{abstract}
Resumen. En el marco de la Teoría de las Metas de Logro y haciendo alusión a la Teoría de la Autodeterminación, el objetivo principal de este trabajo ha consistido en predecir la satisfacción con la vida de los alumnos de educación física (EF) a partir de la percepción del clima motivacional de la clase y actuando como mediadora la orientación de metas de logro. Para cumplir este objetivo, 608 alumnos españoles de Educación Secundaria y $1^{\circ}$ de Bachillerato, con edades comprendidas entre los 12 y los 17 años ( $M=14.51, D T=1.46$; 55.3\% hombres y 44.7\% mujeres) completaron una escala de clima motivacional en EF, una escala de orientación de metas de logro 2 x 2, y una escala de satisfacción con la vida. Para el análisis de los datos se desarrollaron diversos modelos de ecuaciones estructurales con factores latentes. Los principales resultados mostraron una relación significativa y positiva entre el clima de maestría y la aproximación a la maestría y ésta predijo positivamente la satisfacción con la vida. Por otro lado, el clima de rendimiento se relacionó positivamente con la aproximación al rendimiento y ésta predijo negativamente la satisfacción con la vida de los alumnos. Se discuten los resultados en relación con los planteamientos de la psicología positiva de cara a proponer a los profesores ciertas estrategias pedagógicas en las clases de EF.

Palabras clave: Motivación, bienestar subjetivo, psicología positiva, Educación Secundaria, Bachillerato.
\end{abstract}

Abstract. Under the framework of the Achievement Goal Theory and in relation with Self-Determination Theory, the main objective of this work was to predict life satisfaction of physical education (PE) students as a mediator of achievement goals orientation, by means of assessing their perception of in-class motivational climate. A sample of 608 Spanish students of secondary and high school, aged 12 to 17 years old ( $M=14.51, D T$ = 1.46; 55.3\% men, 44.7\% women) completed a motivational climate in PE scale, an achievement goals orientation 2 x 2 scale, and a satisfaction with life scale. Data analyses consisted of several structural equation models with latent factors. Main results showed a significant and positive relationship between mastery climate and mastery approach orientation, which in turn significantly predicted satisfaction with life. Performance climate was positively related to performance approach orientation, which in turn negatively predicted students' life satisfaction. Results are discussed in relation to the approach of positive psychology in order to propose teachers the use of specific pedagogical strategies in PE classes.

Key words: Motivation, subjective well-being, positive psychology, secondary school, high school.

\section{Introducción}

Numerosos informes de investigación (Centers for Disease Control and Prevention, 2011; Ortega et al., 2011; Yeung, Yuan, Hui \& Ferresu, 2016) han resaltado que los niveles de actividad física de los jóvenes en los países industrializados están por debajo de lo considerado necesario para disfrutar de una buena salud y bienestar subjetivo. Por ello, investigadores, profesionales de la salud y responsables políticos han tratado de comprender mejor los factores que influyen en la participación de los jóvenes (Franco, Coterón, Gómez, Brito, \& Martínez, 2017; Granero-Gallegos, Baena-Extremera, Pérez-Quero, OrtizCamacho \& Bracho-Amador, 2014). A pesar de que los antecedentes de la participación en actividades físicas son muy complejos, el rol que desempeña la motivación es, sin duda, una vía evidente e importante para la investigación empírica y aplicada (Standage \& Gillison, 2007). Diversos trabajos se han centrado en el papel que la educación física (EF) juega en el apoyo a las experiencias motivacionales de los alumnos y en favor de su bienestar general (Bagoien, Halvari \& Nesheim, 2010; Taylor, Spray \& Pearson, 2014). Sin embargo, parecen aún insuficientes los estudios empíricos desarrollados para conocer si la motivación en el contexto de la EF influye sobre la motivación e implicación en la práctica física fuera del horario escolar (Taylor, et al., 2014). Por otra parte, las investigaciones que se desarrollen desde esta perspectiva aportarán luz a los efectos que los procesos motivacionales relacionados con la práctica física pueden ejercer sobre marcadores del bienestar de los adolescentes, tales como la satisfacción con la vida (Gunnell, Crocker, Mack, Wilson \& Zumbo, 2014; Standage, Gillison, Ntoumanis \& Treasure, 2012).

Fecha recepción: 06-04-16. Fecha de aceptación: 05-09-16 Melchor Gutiérrez melchor.gutierrez@uv.es
En las últimas décadas, dos han sido las principales teorías que más han facilitado la comprensión de los procesos motivacionales en el contexto de la EF escolar y de la práctica física fuera de la escuela: La Teoría de las Metas de Logro-TML (Ames, 1992; Biddle, 2001; Duda, 2005; Elliot, 2005; Nicholls, 1989; Roberts, 2001) y la Teoría de la Autodeterminación-TAD (Deci \& Ryan, 1985, 2008a; Ryan \& Deci, 2000, 2002; Vansteenkiste, Niemiec \& Soenens, 2010). Tanto la TAD como la TML se pueden utilizar para comprender los factores interpersonales y de motivación que ayudan a explicar las autoevaluaciones de los alumnos, así como su estado de bienestar (Bagoien, et al., 2010; Galand, Boudrenghien \& Rose, 2012; Gunnell, et al., 2014; Jaakkola, Ntoumanis \& Liukkonen, 2016; Standage, et al., 2012; Taylor, et al., 2014).

La TML (Nicholls, 1989) proporciona un enfoque cognitivosocial para estudiar y entender la motivación. De acuerdo con la TML, el principal motivo de las personas en los contextos de logro es desarrollar su competencia. Según la TML, una persona está orientada al ego (otros autores lo denominan rendimiento, comparación) cuando utiliza referencias normativas para evaluar su éxito y competencia. Es decir, esa persona sólo se considera con éxito si supera a los demás. Las personas orientadas a la tarea (también denominado por otros autores maestría, aprendizaje) utilizan referencias personales para evaluar su éxito o fracaso. Es decir, esas personas consideran que tienen éxito y se sienten competentes si mejoran cada día con respecto a sí mismas, sin importarles el rendimiento de los demás (Ames, 1992). Suele ser habitual que una persona se sienta más orientada hacia una meta u otra, pero también es posible que esté simultáneamente orientada tanto al ego como a la tarea, porque las metas de logro poseen carácter ortogonal (Roberts, 2001), es decir, pueden darse simultáneamente, no son excluyentes.

Aunque tradicionalmente se ha considerado la orientación disposicional o meta de logro de las personas desde un modelo dicotómico (orientación al ego, orientación a la tarea), también se han presentado otros modelos teóricos que tratan de explicar 
estas orientaciones disposicionales en los contextos de logro. Éste es el caso de Elliot (1999; 2005), que ha desarrollado un modelo 2 x 2 como alternativa al modelo dicotómico de orientación de metas utilizado durante las dos últimas décadas. A partir de este modelo, las dos tradicionales metas dan lugar a cuatro tipos: de aproximación a la maestría, de evitación de la maestría, de aproximación al rendimiento y de evitación del rendimiento. Diferentes trabajos han mostrado la utilidad de aplicar los constructos de aproximación y evitación a las metas de logro, ya que las personas pueden tener como meta tanto demostrar su competencia como evitar mostrar su incompetencia (Elliot \& McGregor, 2001).

Otro concepto importante en la TML es el clima motivacional creado por los otros significativos (Ames, 1992; Nicholls, 1989; Roberts, 2001), distinguiendo entre climas de maestría (aprendizaje) y climas de rendimiento (comparación), en el contexto de la clase o el entrenamiento deportivo (Biddle, 2001). Los climas de maestría se refieren a cómo los otros significativos (profesores, entrenadores, padres) estructuran el ambiente. El clima de maestría en EF se caracteriza por la promoción de aprendizaje y apoyo de los profesores y la percepción de los alumnos de una atmósfera de cooperación y ayuda donde el esfuerzo es importante para mejorar. Por el contrario, el clima de rendimiento se caracteriza por la promoción de competición por parte de los profesores, la comparación normativa de los estudiantes y la rivalidad entre ellos, así como la perspectiva de que los errores son castigados, por lo cual los alumnos pueden tener miedo a cometerlos (Biddle, 2001; Roberts, 2001).

De acuerdo con la TML, el clima motivacional situacional y las metas de logro disposicionales interactúan para producir consecuencias afectivas, cognitivas y conductuales en las personas (Ames, 1992; Nicholls, 1989). Diversas investigaciones apoyan la existencia de relaciones significativas entre los climas motivacionales y las orientaciones de meta de los alumnos (Jaakkola, et al., 2016; Ruiz-Juan \& Baena-Extremera, 2015; Smith, Smoll \& Cumming, 2009). Reinboth \& Duda (2006) destacan el efecto del entorno o clima motivacional percibido en el que el individuo muestra su competencia, sobre las orientaciones motivacionales subsiguientes. Habitualmente, la percepción de los alumnos sobre el clima de aprendizaje aparece más fuertemente correlacionada con la meta orientada a la maestría, mientras que la percepción del clima de comparación se asocia con la orientación de aproximación al rendimiento y de evitación del rendimiento (Granero-Gallegos \& Baena-Extremera, 2014; Halvari, Skjesol \& Bagoien, 2011; Ommundsen, 2006), sobre todo en los contextos escolares, más que en los contextos competitivos. La TML indica que la adherencia a la práctica física puede ser facilitada por ambientes de aprendizaje que enfaticen la mejora y el progreso personal, objetivo principal del clima de maestría, coherente con el espíritu de la psicología positiva (Church, Elliot \& Gable, 2001; Isorna, Rial \& Vaquero, 2014).

También la TAD ha sido ampliamente empleada para explorar la motivación de los alumnos en el contexto escolar (Amado, Sánchez-Miguel, Leo, Sánchez-Oliva \& García-Calvo, 2014). La TAD contempla diferentes tipos de motivación que varían según sus niveles de autodeterminación a lo largo de un continuo. En el extremo más auto-determinado o autónomo se encuentra la motivación intrínseca, que se refiere a la práctica de una actividad por sí misma, es decir, porque es placentera, divertida e interesante. A continuación se encuentran tres tipos de motivación extrínseca, lo que refleja el compromiso con la práctica por razones ajenas a la propia actividad. En orden descendente de auto-determinación, los diferentes motivos son: regulación identificada (práctica de la actividad para obtener resultados deseados y valorados personalmente), regulación introyectada (participar en la práctica por sentirse digno o por evitar sentimientos de culpa o vergüenza), y regulación externa (participar por obtener una recompensa o evitar un castigo). Cuando una persona no encuentra ninguna razón por la que valga la pena la práctica física, se dice que no está ni intrínseca ni extrínsecamente motivada, simplemente está desmotivada (Deci \& Ryan, 2000, 2008b).

Como fundamento de la TAD, se postula que los seres humanos tienen tres necesidades psicológicas básicas (autonomía, competencia, relación) que han de satisfacer, y cuyo cumplimiento aumenta su bienestar. La autonomía se caracteriza por sentimientos de acción personal guiados por la voluntad propia (autodeterminación); la competencia se caracteriza por sentimientos de eficacia para realizar tareas desafiantes de manera óptima; y la relación se caracteriza por sentimientos de conexión significativa con los demás (Ryan \& Deci, 2002).

Las escuelas han sido reconocidas como contextos clave no sólo para promover la práctica de actividad física y la promoción de estilos de vida saludables, sino también para ayudar a los jóvenes a desarrollar todo su potencial y sentirse bien consigo mismos (Centros para el Control de Enfermedades y Prevención, 2011). Existe, por tanto, un fuerte interés por investigar los procesos motivacionales que intervienen en el entorno escolar para comprender las relaciones entre la actividad física y el bienestar de los alumnos (Taylor, et al., 2014).

El bienestar se refiere a un «funcionamiento psicológico óptimo» (Ryan \& Deci 2001, p.142). Puede definirse como el estado de sentirse a gusto, feliz o próspero. Según Lu \& Buchanan (2014), el bienestar está relacionado con el potencial de un individuo para tener una vida feliz y saludable, y en este sentido la EF puede considerarse un contexto apropiado para desarrollar tales características. Algunas investigaciones han sugerido que los alumnos con emociones positivas es más probable que desarrollen un estilo de vida activo, lo cual es uno de los fines principales de la EF (Rink, 2010), y para ello el clima motivacional de la clase puede resultar determinante a la hora de generar el bienestar que los alumnos necesitan para sentirse seguros, apoyados y reconocidos, es decir, para progresar como personas y sentirse mentalmente saludables (Sistkos, Papaioannou \& Propios, 2011).

El bienestar subjetivo se define como «una amplia categoría de fenómenos que incluye las respuestas emocionales de las personas, la satisfacción con los dominios, y los juicios globales sobre la satisfacción con la vida» (Diener, Suh, Lucas \& Smith, 1999, p. 277). Se distinguen dos aspectos principales en el bienestar subjetivo, el afectivo y el cognitivo. El dominio afectivo se refiere a las emociones positivas y negativas, mientras que el cognitivo hace referencia a la satisfacción con la vida (Diener \& Diener, 2009). En la adolescencia, la satisfacción con la vida puede ser un indicador clave de la salud mental y del bienestar subjetivo (Anataramian, Huebner \& Valois, 2008). Pero Elmore \& Huebner (2010) señalan que, a pesar de reconocer su importancia en la investigación psicológica, la satisfacción con la vida ha sido una variable frecuentemente abordada en personas adultas y mayores pero menos estudiada con muestras de adolescentes.

En el marco de la motivación autodeterminada, Bagoien, Halvari \& Nesheim (2010) afirman que el concepto de bienestar subjetivo, en general, está indicado por la presencia de vitalidad, el afecto positivo y la satisfacción con la vida de los estudiantes, y que está positivamente asociado con la satisfacción de las necesidades psicológicas básicas, la motivación autónoma, la percepción de competencia y la práctica de actividad física. Otros investigadores han concluido que los indicadores del bienestar subjetivo están positivamente correlacionados tanto con la satisfacción de las necesidades básicas (Wilson, Rodgers, Fraser, Murray \& McIntyre, 2004) como con la motivación hacia el ejercicio (Georgiadis, Biddle \& Chatzisarantis, 2001), y 
que la participación en actividad física se encuentra positivamente asociada con diversos indicadores del bienestar subjetivo, la reducción de la depresión y la ansiedad (Long \& van Stavel, 1999), y un cambio positivo en la satisfacción con la vida, la vitalidad y la autoestima (Fox, 1997; Reigal, Videra \& Gil, 2014; Ryan \& Deci, 2000).

Los resultados que Standage, et al. (2012) encontraron en su trabajo de investigación proporcionan apoyo empírico a la relación entre los procesos de motivación que tienen lugar en los contextos de EF y los índices de salud relacionados con el bienestar de los alumnos. Estos resultados también refuerzan la idea de los beneficios motivacionales que los alumnos experimentan derivados de sus interacciones basadas en el apoyo a la autonomía por parte de sus profesores, los cuales proveen las condiciones sociales conducentes a la satisfacción de las necesidades básicas y al bienestar subjetivo.

Por su parte, Brown \& Fry (2014) encontraron una relación positiva entre el clima motivacional positivo y la satisfacción con la vida. Los alumnos que percibieron un clima de maestría en su práctica física informaron de una mayor satisfacción con la vida. Adicionalmente, Galand, et al. (2012) encontraron una asociación entre el clima de maestría y la satisfacción con la vida, actuando como variable mediadora la orientación a la tarea. Por tanto, resulta de interés estudiar la posible influencia de los climas motivacionales en EF sobre las orientaciones de meta de los alumnos y los resultados que de ello puedan derivarse.

Fundamentados en el anterior marco conceptual, el objetivo de este trabajo ha consistido en analizar las relaciones entre la percepción del clima motivacional de la clase de EF, la orientación de metas de logro de los alumnos y su satisfacción con la vida (Fig. 1). Partimos de las siguientes hipótesis: (a) Que la percepción del clima de maestría estaría directamente relacionada con la meta orientada a la maestría, mientras que la percepción de un clima de rendimiento se relacionaría positivamente con la meta de orientación al rendimiento; (b) Que la orientación de metas de logro de los alumnos actuaría como mediador entre la percepción del clima motivacional de la clase y la satisfacción con la vida de los alumnos; (c) que la orientación a la maestría estaría positivamente relacionada, mientras que la orientación al rendimiento lo estaría negativamente, con la satisfacción con la vida de los alumnos de EF.

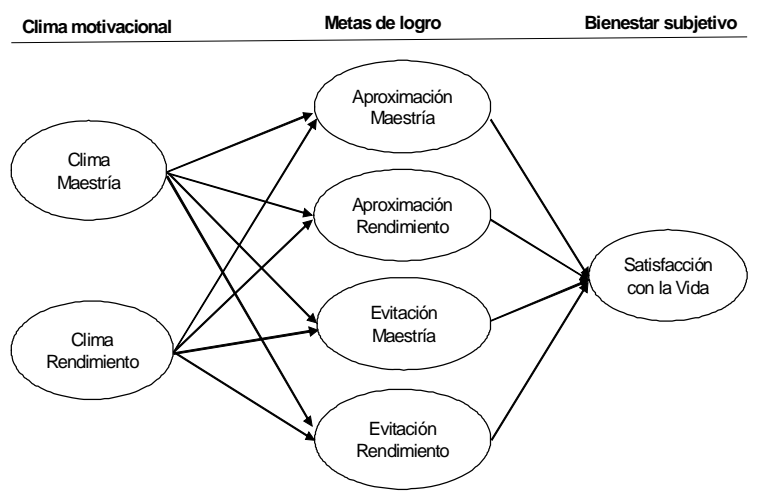

Fig. 1. Modelo teórico propuesto para las relaciones entre los factores del clima motivaciona de la clase de EF, la orientación de metas de logro y la satisfacción con la vida de los alumnos.

\section{Método}

\section{Participantes}

Los participantes han sido 608 alumnos de Educación Secundaria y $1^{\circ}$ de Bachillerato (336 chicos y 272 chicas), con edades comprendidas entre los 12 y los 17 años $(M=14.51, D T$ =1.46), distribuidos por sexo y curso académico según muestra la Tabla 1. La selección de los participantes fue incidental o por conveniencia y se desarrolló por el método de colaboradores, procurando que fuesen representativos de la población adolescente española y que su distribución en cuanto a sexo y curso fuese equilibrada. A partir de un curso nacional de perfeccionamiento en el que participaron 48 profesores de educación física y técnicos deportivos, se les solicitó colaboración para aplicar los instrumentos en sus grupos de trabajo. Además, se les propuso que si tenían conocimiento de otros compañeros que pudieran también colaborar nos lo indicaran, iniciando así un muestreo por bola de nieve (snowball sampling). De todos los que mostraron interés por colaborar en la investigación, se tomaron los que aportaban grupos de edad y practicantes de actividad física suficientemente diversos como para conseguir que la muestra final de estudio fuese heterogénea y lo más cercana posible a la que se hubiese obtenido aleatoriamente.

Tabla 1

\begin{tabular}{|c|c|c|c|c|c|c|}
\hline \multirow{2}{*}{$\begin{array}{c}\text { Curso } \\
1^{\circ} \mathrm{ESO}\end{array}$} & \multicolumn{2}{|c|}{ Chicos } & \multicolumn{2}{|c|}{ Chicas } & \multicolumn{2}{|c|}{ Total } \\
\hline & 64 & $10.5 \%$ & 51 & $8.4 \%$ & 115 & $18.9 \%$ \\
\hline $2^{\circ} \mathrm{ESO}$ & 77 & $12.7 \%$ & 60 & $9.9 \%$ & 137 & $22.5 \%$ \\
\hline $3^{\circ} \mathrm{ESO}$ & 73 & $20.0 \%$ & 60 & $9.9 \%$ & 133 & $21.9 \%$ \\
\hline $4^{\circ} \mathrm{ESO}$ & 70 & $11.5 \%$ & 50 & $8.2 \%$ & 120 & $19.7 \%$ \\
\hline $1^{\circ} \mathrm{BACH}$. & 52 & $8.6 \%$ & 51 & $8.4 \%$ & 103 & $16.9 \%$ \\
\hline Total & 336 & $55.3 \%$ & 272 & $44.7 \%$ & 608 & $100.0 \%$ \\
\hline
\end{tabular}

\section{Instrumentos}

Clima motivacional de la clase de EF. Se empleó la versión española de la Perception of Motivational Climate Scale, de Biddle, et al. (1995), validada al contexto español por Gutiérrez \& Ruiz (2009). Tal como muestran sus autores, esta escala consta de 19 ítems agrupados en cinco factores: Búsqueda de progreso por los alumnos (ej.: Los alumnos aprenden nuevas habilidades y se sienten satisfechos), Promoción de aprendizaje por el profesor (ej.: El profesor/a de EF está satisfecho/a cuando cada alumno aprende cosas nuevas), Búsqueda de comparación por los alumnos (ej.: Los alumnos intentan hacer las cosas mejor que los demás), Promoción de comparación por el profesor (El profesor/a sólo se ocupa de quienes hacen bien los ejercicios) y Miedo de los alumnos a cometer errores (ej.: Los alumnos tienen miedo a cometer errores). Los dos primeros factores se agrupan en un factor de segundo orden denominado Aprendizaje o Maestría, y los tres últimos en el factor Comparación, Ejecución, o Rendimiento, según diferentes autores.

Tanto Gutiérrez \& Ruiz (2009) como Gutiérrez (2014) desarrollaron sucesivas comprobaciones de la adecuación de esta escala a muestras de adolescentes españoles, y mediante análisis factoriales confirmatorios obtuvieron la misma estructura que Biddle, et al. (1995). La consistencia interna (coeficientes alfa de Cronbach) para cada uno de los factores obtenida por Gutiérrez (2014) es la siguiente: Búsqueda de progreso por los alumnos $(\alpha=.93)$, Promoción de aprendizaje por el profesor ( $\alpha=.85)$, Búsqueda de comparación por los alumnos $(\alpha=.77)$, Promoción de comparación por el profesor $(\alpha=.82)$, Miedo de los alumnos a cometer errores $(\alpha=.85)$, Aprendizaje o Maestría $(\alpha=.92)$ y Comparación, Ejecución, o Rendimiento $(\alpha=$ $.91)$.

Los coeficientes de consistencia interna (alfas) obtenidos en los cinco factores para la presente muestra han sido, respectivamente: .83, .83, .70, .74, .69. En cuanto a los factores de segundo orden su fiabilidad ha sido: Clima de Maestría $\alpha=.89$ y Clima de Rendimiento $\alpha=.81$.

Orientación de metas de logro en EF. Se ha utilizado la Escala de Metas de Logro en EF 2X2, que procede de los trabajos de Elliot \& McGregor (2001), adaptada por Guan, Xiang, McBride \& Burne (2006) al contexto de la EF. En este trabajo se empleó una adaptación española de Moreno, González-Cutre \& Sicilia (2008). Está formado por 12 ítems agrupados en cuatro dimensiones: Aproximación a la Maestría (ej., «Es importante para mi entender el contenido de la asignatura tan a fondo 
como sea posible»), Evitación de la Maestría (ej., «A menudo me preocupa no poder aprender todo lo que hay que aprender»), Aproximación al Rendimiento (ej., «Mi objetivo es conseguir un mayor nivel que la mayoría de los estudiantes), y Evitación del Rendimiento (ej., «Mi objetivo es evitar hacerlo mal»). Moreno, et al. (2008) obtuvieron valores de fiabilidad de $.70, .71, .67$ y .72, respectivamente. Los coeficientes alfa obtenidos con esta muestra de estudio han sido, respectivamente: .82, .73, .72, .69.

Bienestar subjetivo. Se empleó la Satisfaction with Life ScaleSWLS (Diener, Emmons, Larsen \& Griffin, 1985), adaptación al castellano de Atienza, Pons, Balaguer \& García-Merita (2000) con muestras adolescentes. Esta escala ha aportado evidencias de validez y fiabilidad en numerosos países y con muestras de diferentes edades y condiciones sociales, por ejemplo la de Atienza, Balaguer, Corte-Real \& Fonseca (2016) con adolescentes españoles y portugueses, y la de Núñez, Martín-Albo \& Domínguez (2010) con muestras de adultos españoles practicantes de actividad física. Está compuesta por cinco ítems, cuyo objetivo es proporcionar un índice general de satisfacción con la vida referido a la percepción de bienestar subjetivo (ejemplos: «Estoy satisfecho con mi vida»; «En la mayoría de los aspectos mi vida es como quiero que sea»). La consistencia interna de esta escala para la presente muestra ha sido de $\alpha=.81$.

En todos los instrumentos utilizados, los alumnos debían indicar su grado de acuerdo con cada una de las expresiones en una escala tipo Likert con cinco alternativas, desde (1) totalmente en desacuerdo, hasta (5) totalmente de acuerdo.

\section{Procedimiento}

Tras obtener la aprobación del personal directivo de los centros educativos y deportivos, se enviaron cartas a los padres de los alumnos solicitándoles permiso para la participación de sus hijos, por ser éstos menores de edad. Sólo los alumnos que informaron de la autorización expresa de sus padres o tutores legales tomaron parte en el estudio. Todos los alumnos que participaron lo hicieron voluntariamente. Los instrumentos fueron aplicados en diversas instalaciones deportivas y en diferentes centros españoles de Educación Secundaria y Bachillerato. Para garantizar la confidencialidad de los datos, todos los instrumentos eran anónimos y fueron aplicados por un miembro del equipo de investigación, sin la presencia de los profesores y técnicos, con objeto de facilitar la espontaneidad de los participantes. El tiempo invertido por los alumnos-deportistas para responder las escalas no superó los 15 minutos.

\section{Análisis estadísticos}

Aparte de calcular la consistencia interna (alfa de Cronbach) de cada una de las dimensiones de los instrumentos utilizados para la recogida de datos, se calcularon las correlaciones entre todas las variables estudiadas. Seguidamente, se sometió a verificación, mediante Modelos de Ecuaciones Estructurales con factores latentes, el modelo teórico que se muestra en la Fig. 1.

Los modelos confirmatorios se han estimado mediante WLSMV (Weighted Least Squares Means and Variances corrected), dada la falta de normalidad de las variables y la naturaleza ordinal de los ítems de las escalas (Finney \& DiStefano, 2006). El ajuste de los modelos se ha evaluado utilizando los criterios habitualmente recomendados en la literatura y disponibles para este tipo de estimación (Hu \& Bentler, 1999). Concretamente, los estadísticos e índices de ajuste utilizados son: (a) estadístico $X^{2}$ (Kaplan, 2000); (b) Comparative Fit Index (CFI; Bentler, 1990) mayor de .90 (e idealmente mayor de .95; Hu \& Bentler, 1999); y (c) Root Mean Square Error of Approximation (RMSEA), cuyos valores son aceptables cuando son iguales o menores a .08, y excelentes si son menores de .05. Adicionalmente se han calculado los efectos indirectos. Para ello se ha empleado remuestreo bootstrap con intervalos de confianza con el sesgo corregido (bias corrected confidence intervals), el procedimiento recomendado por la literatura especializada (MacKinnon, 2008).

\section{Resultados}

\section{Resultados descriptivos}

Tal como muestra la Tabla 2, las puntuaciones más altas otorgadas por los alumnos han correspondido a la percepción de promoción del aprendizaje por el profesor $(M=3.54, D T=$ $0.95)$, la orientación de meta de aproximación a la maestría $(M=$ $3.44, D T=0.98)$ y la satisfacción con la vida $(M=3.60, D T=$ $0.76)$. Las variables a las que se les ha otorgado menor puntuación han sido la percepción de promoción de comparación por el profesor $(M=2.72, D T=0.82)$ y el miedo a cometer errores $(M=2.82, D T=0.82)$. En cuanto a la fiabilidad de los instrumentos utilizados, todos ellos han mostrado una buena consistencia interna excepto dos factores, el del clima de promoción de comparación por el profesor y el de la orientación de evitación del rendimiento que han obtenido valores de alfa igual a .69, próximos al .70 habitualmente reconocido como satisfactorio, según recomendación de Nunnally (1978). Una razón para este menor nivel de los alfa puede ser que ambos factores están formados únicamente por tres ítems cada uno.

Con respecto a las relaciones entre las variables estudiadas, cabe destacar, aparte de las lógicas correlaciones elevadas entre la búsqueda de progreso por los alumnos y la promoción de aprendizaje por el profesor con el clima de maestría (como factor de segundo orden agrupador de las dos variables anteriores), la relación positiva de la búsqueda de progreso por los alumnos con la aproximación a la maestría $(r=.69, \mathrm{p}<.01)$, la relación del clima de maestría con la orientación de meta de aproximación a la maestría $(\mathrm{r}=.68, \mathrm{p}<.01)$ y con la satisfacción con la vida $(r=.62, p<.01)$, el clima de rendimiento con la evitación del rendimiento $(\mathrm{r}=.50, \mathrm{p}<.01)$, la aproximación a la maestría con la aproximación al rendimiento $(\mathrm{r}=.56, \mathrm{p}<.01)$ y con la satisfacción con la vida $(\mathrm{r}=.50, \mathrm{p}<.01)$, y la evitación de la maestría con la evitación del rendimiento $(\mathrm{r}=.56, \mathrm{p}<.01)$.

Tabla 2.

Medias, desviaciones típicas, alfas y correlaciones bivariadas entre las variables estudiadas \begin{tabular}{llllllllllllll} 
Medias, desviaciones típicas, alfas y correlaciones bivariadas entre las variables estudiadas \\
\hline Variables & $\mathbf{1}$ & $\mathbf{2}$ & $\mathbf{3}$ & $\mathbf{4}$ & $\mathbf{5}$ & $\mathbf{6}$ & $\mathbf{7}$ & $\mathbf{8}$ & $\mathbf{9}$ & $\mathbf{1 0}$ & $\mathbf{1 1}$ & $\mathbf{1 2}$
\end{tabular} \begin{tabular}{lcccccccccccc} 
1.BusqPrAl & 1 & $\mathbf{2}$ & $\mathbf{3}$ & $\mathbf{4}$ & $\mathbf{5}$ & $\mathbf{6}$ & $\mathbf{7}$ & $\mathbf{8}$ & $\mathbf{9}$ & $\mathbf{1 0}$ & $\mathbf{1 1}$ & $\mathbf{1 2}$ \\
\hline & & .65 & .27 & .42 & .89 & .55 & .69 & .48 & .41 & .57 & .60
\end{tabular}

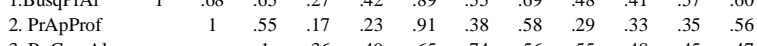
$\begin{array}{lllllllllll}\text { 3. BuComAl } & 1 & .36 & .49 & .65 & .74 & .56 & .55 & .48 & .45 & .47\end{array}$ $\begin{array}{llllllllll}\text { 4. PrComProf } & 1 & .57 & .22 & .78 & .21 & .32 & .26 & .36 & .19\end{array}$ $\begin{array}{lcccccccc}\text { 5. MiedoErr } & 1 & .33 & .89 & .31 & .39 & .32 & .40 & .028 \\ \text { 6. ClMaestría } & 1 & .32 & .38 & .68 & .38 & .39 & .42 & .62\end{array}$ 7. ClRendim.

8. AprMaest.

9. EvMaestría

9. EvMaestría

10. AprRenc \begin{tabular}{llllllllllllc}
12. SalVida & & & & & & & & & & & & \\
\hline$M$ & 3.19 & 3.54 & 3.33 & 2.72 & 2.82 & 3.39 & 2.94 & 3.44 & 2.91 & 3.10 & 3.08 & 3.60
\end{tabular} $\begin{array}{lllllllllllll}D T & 0.86 & 0.95 & 0.82 & 0.82 & 0.82 & 0.85 & 0.67 & 0.98 & 0.89 & 0.92 & 0.90 & 0.76\end{array}$

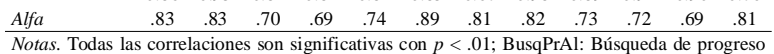
por los alumnos; PrApProf: Promoción de aprendizaje por el profesor; BuComAl: Búsqueda de comparación por los alumnos; PrComProf: Promoción de comparación por el profesor; MiedoErr: Miedo de los alumnos a cometer errores; ClMaestría: Clima de Maestría; ClRendim.: Clima de rendimiento; AprMaest: Aproximacoión a la mestrí; EvMaestría: Evitación de la Clina de maestria; AprRend.: Aproximacion

Predicción de la satisfacción con la vida de los alumnos

El modelo hipotético inicial planteaba que la percepción del clima motivacional de la clase sería capaz de predecir la orientación de metas de logro la cual, a su vez, tendría capacidad predictiva sobre la satisfacción con la vida de los alumnos. Este modelo general a-priori se puso a prueba mediante ecuaciones estructurales, obteniendo los siguientes índices de ajuste: $X^{2}{ }_{190}$ $=660.12, p<.001, \mathrm{CFI}=.85$, RMSEA $=.073[$ IC $90 \% .067-$ .079] (Fig. 2), 


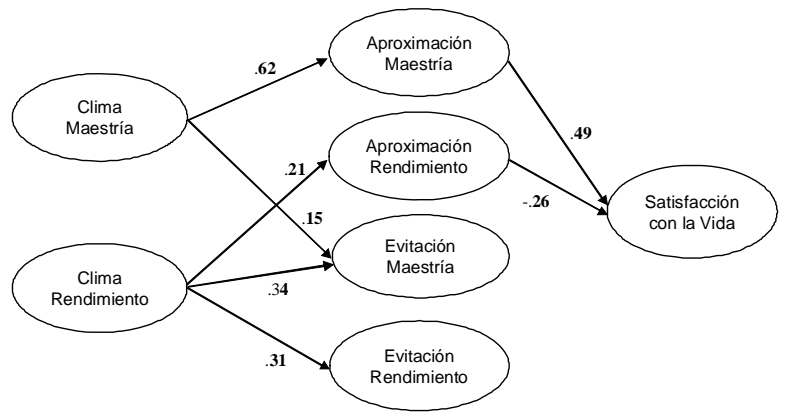

Fig. 2. Solución estandarizada para el modelo propuesto de las relaciones entre el clima motivacional de la clase de EF, la orientación de metas de logro y la satisfacción con la vida de los alumnos. Nota: por simplicidad, solamente se muestran las relaciones estructurales entre variables latentes y no el modelo de medida.

Dado que los resultados de este modelo no pueden considerarse satisfactorios, se probó un nuevo modelo (Fig. 3) en el que se eliminaron los factores de evitación que no aportaban capacidad predictiva sobre la satisfacción con la vida y se añadió una saturación cruzada de la búsqueda de comparación por los alumnos en el clima de maestría sugerida por los índices de modificación. El ajuste a los datos obtenido fue mucho más satisfactorio: $X_{95}^{2}=252.23, \mathrm{p}<.001$, CFI $=.93$, RMSEA $=.062[.053-$ $.072]$.

Aunque por simplicidad, la Figura 3 no representa el modelo de medida de los factores latentes, éste era satisfactorio. Todas las saturaciones de los indicadores del clima motivacional estaban entre .53 y .79, los de aproximación entre .62 y .81 y los de satisfacción con la vida entre .53 y .79. Los resultados muestran que el clima de maestría se encuentra positiva y directamente relacionado con la orientación de aproximación a la maestría y con la aproximación al rendimiento, mientras que el clima de rendimiento está relacionado positivamente con la aproximación al rendimiento. Por otro lado, la aproximación a la maestría se relaciona positivamente con la satisfacción con la vida y, a su vez, la aproximación al rendimiento muestra una relación negativa con la satisfacción con la vida de los alumnos. La orientación de metas de aproximación a la maestría es predicha en un $50.1 \%$ por el clima de maestría ( $\beta=.71$ ), la aproximación al rendimiento es predicha en un $10.9 \%$ por el clima de maestría $(\beta=.18)$ y por el clima de rendimiento $(\beta=.29)$. Finalmente, la satisfacción con la vida de los alumnos es predicha en un $24.2 \%$ por la aproximación a la maestría $(\beta=.62)$ y de manera inversa por la aproximación al rendimiento $(\beta=-.49)$.

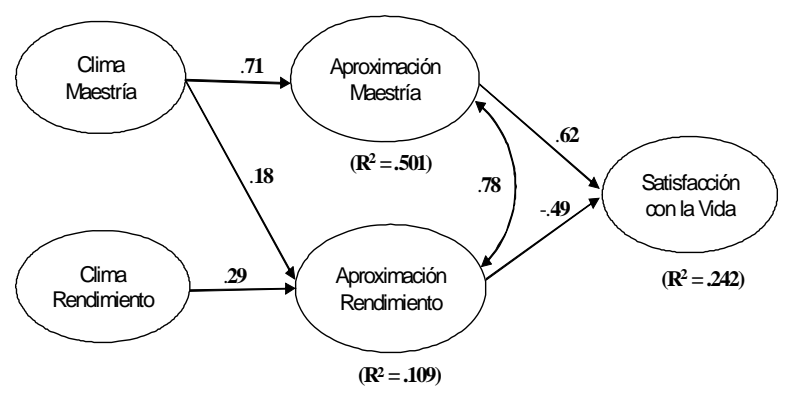

Fig. 3. Resultado final de la solución estandarizada para el modelo propuesto de predicción de la satisfacción con la vida de los alumnos de EF, a partir del clima motivacional de la clase y de las orientaciones de meta. Nota: por simplicidad, solamente se muestran las relaciones estructurales entre variables latentes y no el modelo de medida.

El modelo estructural permite calcular los efectos indirectos de los dos factores de clima sobre la satisfacción con la vida. El clima de maestría presenta dos efectos indirectos sobre la satisfacción, el mediado por la aproximación al rendimiento, que resultó no significativo ( $\beta=-.08, \mathrm{p}=.147)$, y el mediado por la aproximación a la maestría que sí resultó estadísticamente significativo y elevado $(\beta=.44, \mathrm{p}<.001)$. Por su parte, el clima de rendimiento presentó también dos efectos indirectos, el mediado por la aproximación a la maestría, no significativo $(\beta=$ $.001, \mathrm{p}=.995)$, y el mediado por la aproximación al rendimiento que sí resultó significativo $(\beta=-.14, \mathrm{p}=.007)$. Puede verse que los efectos indirectos del clima de maestría y rendimiento fluyen a través de las correspondientes aproximaciones, y que es un efecto positivo sobre la satisfacción en el caso del clima de maestría, pero negativo en el caso del clima de rendimiento.

\section{Discusión y conclusiones}

El objetivo principal de este trabajo ha consistido en la aplicación de modelos de ecuaciones estructurales para predecir la satisfacción con la vida de los adolescentes a partir de su percepción del clima motivacional de la clase de EF y de su orientación de metas de logro. Se hipotetizó que la percepción del clima motivacional de la clase estaría relacionada con las orientaciones de meta de los alumnos, y éstas, a su vez, con su satisfacción con la vida.

Cabe destacar, en primer lugar, que la fiabilidad de los instrumentos empleados ha resultado satisfactoria para su empleo en esta muestra de alumnos. Con respecto al cumplimiento de las hipótesis planteadas, cabe señalar que se han cumplido en su mayor parte, aunque con algunas matizaciones que seguidamente indicaremos.

En cuanto a la primera hipótesis, que suponía el efecto directo de la percepción del clima de maestría sobre la meta orientada a la maestría, y de la percepción del clima de rendimiento sobre la meta de orientación al rendimiento, ésta se ha visto cumplida. Estos resultados se corresponden con los obtenidos por Ommundsen (2006), Smith, et al. (2009), Halvari, et al. (2011), Granero-Gallegos \& Baena-Extremera (2014), y Jaakkola, et al. (2016), en todos los cuales se muestra una relación directa entre los climas motivacionales y las orientaciones de meta de los alumnos en las clases de EF y en el contexto de la práctica deportiva.

En relación con la segunda hipótesis, según la cual se esperaba que la orientación de metas actuaría como mediador entre la percepción del clima motivacional de la clase y la satisfacción con la vida de los alumnos, ésta se ha visto cumplida, aunque sólo en la vertiente de aproximación a la maestría y al rendimiento, no en las dimensiones de evitación de la maestría y evitación del rendimiento. Una interpretación que damos a estos resultados es que los alumnos han considerado las orientaciones de meta en sentido tradicional, contemplando la orientación a la maestría y la orientación al rendimiento, y no han tenido en cuenta las otras dos dimensiones (evitación de la maestría y evitación del rendimiento) que ofrece el modelo 2 x 2 de Elliot \& McGregor (2001). Es posible que en contextos de logro con mayor necesidad de comparación, como la práctica deportiva competitiva, funcione adecuadamente el modelo de metas 2 $\mathrm{x} 2$, sin embargo, en el contexto de la EF, más educativo y menos necesitado de la comparación y la supremacía de unos sobre otros, las dos orientaciones tradicionales de la TML se ajustan mejor a los intereses de los alumnos, al menos a criterio de la muestra estudiada. Apoyo a los resultados parcialmente cumplidos de esta hipótesis los encontramos en el trabajo de Church, et al. (2001), según los cuales la percepción del clima de clase influencia la adopción de las metas de logro.

Por último, la tercera hipótesis planteaba que la orientación a la maestría estaría positivamente relacionada con la satisfacción con la vida de los alumnos, mientras que la orientación al rendimiento presentaría una relación negativa. Tal como muestran los resultados, según el segundo modelo teórico testado (Fig. 3), se cumple que la aproximación a la maestría predice 
positivamente la satisfacción con la vida, mientras que la aproximación al rendimiento muestra una relación inversa en la predicción de la satisfacción con la vida de los alumnos de EF. Estos resultados se encuentran en línea con los mostrados por la literatura especializada sobre los hallazgos de Galand, et al. (2012), y Gunnell, et al. (2014), quienes analizando la predicción de la satisfacción con la vida de los alumnos encontraron como variable mediadora la orientación a la tarea.

Como conclusión final cabe señalar que el clima de la clase ha mostrado capacidad predictiva sobre la satisfacción con la vida de los alumnos de EF, actuando como mediadoras las orientaciones de meta, siendo el clima de maestría el de mayor capacidad predictiva de la satisfacción con la vida de los alumnos, resultado coincidente con el encontrado en el trabajo de Galand, et al. (2012), y con los obtenidos por Núñez, León, González \& Martín-Albo (2011).

Desde una perspectiva aplicada, estos resultados nos llevan a proponer a los profesionales de la EF la promoción de ambientes de clase en los que predomine el clima de maestría o aprendizaje, dado que es más probable que éste influya sobre la orientación a la maestría de los alumnos y ésta, a su vez, se relacionará positivamente con su satisfacción con la vida. Por el contrario, hemos visto que cuanto mayor sea la orientación al rendimiento menor será la satisfacción con la vida de los alumnos en EF. Aparte de esto, nos hacemos eco de los hallazgos y propuestas de González, Garcés de los Fayos \& García (2012), que encontraron una correspondencia positiva entre los índices de bienestar subjetivo percibido por los alumnos y la forma en que sus docentes transmiten la EF, lo cual les llevó a resaltar la importancia que la transmisión pedagógica de la actividad física puede desempeñar como modelo favorecedor del bienestar subjetivo de los adolescentes. Señalan estos autores la necesidad de establecer medidas para que los estilos educativos de los profesores de EF influyan positivamente en sus alumnos, de tal manera que se consigan mayores niveles de implicación, compromiso y participación. Todo ello incidirá favorablemente en el desarrollo de la personalidad de los alumnos, así como en su bienestar y en la adopción de un estilo de vida activo y autosuficiente, aspectos fundamentales de la psicología positiva (Sun \& Shek, 2012). Además, cabe recordar, como señalaban Taylor, et al. (2014), que las escuelas han sido consideradas lugares apropiados para ayudar a los jóvenes a desarrollar todo su potencial y sentirse bien consigo mismos. Así pues, en las manos de los profesores recae gran parte de la responsabilidad de cumplir este objetivo.

Como cualquier estudio, éste presenta algunas limitaciones. Aunque el tamaño de la muestra es razonablemente amplio, los datos fueron obtenidos a través de colaboradores o muestreo por conveniencia, y recogidos mediante autoinformes de los alumnos, lo cual ha podido incorporar en cierto modo el efecto de la deseabilidad social. Además, los datos utilizados son transversales, por lo que no se pueden establecer conclusiones fundamentadas en relaciones causa-efecto. En este sentido, un diseño longitudinal en el que se tomaran datos en varios momentos temporales proporcionaría mayor garantía a las conclusiones. Por otro lado, el modelo sometido a comprobación mediante ecuaciones estructurales es sólo uno de los posibles, pudiendo existir otros modelos capaces de contribuir con nuevas explicaciones a la interpretación de las relaciones entre las variables estudiadas, o incluir otras no analizadas en este momento. Por ejemplo, como advertían Gallagher \& Vella-Brodrick (2008), podría resultar conveniente contemplar los otros dos componentes del bienestar subjetivo de los adolescentes (el afecto positivo y el afecto negativo), y no limitarse a su representación a través de la satisfacción con la vida, puesto que existen resultados en la literatura científica que indican la predicción diferencial de cada uno de los componentes que integran el bienestar subjetivo de los alumnos.

\section{Referencias}

Amado, D., Sánchez-Miguel, P., Leo, F. M., Sánchez-Oliva, D., \& GarcíaCalvo, T. (2014). Diferencias de género en la motivación y percepción de utilidad del deporte escolar. Revista Internacional de Medicina y Ciencias de la Actividad Física y el Deporte, 14(56), 651-664.

Ames, C. (1992). Classrooms: goals, structures, and student motivation. Journal of Educational Psychology, 84, 261-271.

Anataramian, S. P., Huebner, E. S., \& Valois, R. F. (2008). Adolescent life satisfaction. Applied Psychology, 57, 112-126.

Atienza, F. L., Balaguer, I., Corte-Real, N., \& Fonseca, A. M. (2016). Factorial invariance of the Satisfaction with Life Scale in adolescents from Spain and Portugal. Psicothema, 28(3), 353-358.

Atienza, F. L., Pons, D., Balaguer, I., \& García-Merita, M. (2000). Propiedades psicométricas de la escala de satisfacción con la vida en adolescentes. Psicothema, 12, 314-320.

Bagoien, T. E., Halvari, H., \& Nesheim, H. (2010). Self-determined motivation in physical education and its links to motivation for leisuretime physical activity, and well-being in general. Perceptual and Motor Skills, 111(2), 407-432.

Bentler, P. M. (1990). Comparative fit indices in structural models. Psychological Bulletin, 107, 238-246.

Biddle, S. J. (2001). Enhancing motivation in physical education. En G. C. Roberts (Ed.), Advances in motivation in sport and exercise (pp. 101-127). Champaign, IL: Human Kinetics.

Biddle, S. J, Cury, F., Goudas, M., Sarrazin, Ph., Famose, J.P. y Durand, M. (1995). Development of scales to measure perceived physical education class climate: A cross-national project. British Journal of Educational Psychology, 65, 341-358.

Brown, T .C., \& Fry, M. D. (2014). Motivational climate, staff and members' behaviors, and members' psychological well-being at a national fitness franchise. Research Quarterly for Exercise and Sport, 85, 208-217.

Centers for Disease Control and Prevention. (2011). School health guidelines to promote healthy eating and physical activity. Morbidity and Mortality Weekly Report. Recommendations and Reports. U.S. Department of Health and Human Services.

Church, M. A., Elliot, A. J., \& Gable, S. L. (2001). Perceptions of classroom environment, achievement goals, and achievement outcomes. Journal of Educational Psychology, 93(1), 43-54.

Deci, E. L. \& Ryan, R. M. (1985). Intrinsic motivation and selfdetermination in human behavior. New York: Plenum Press.

Deci, E. L., \& Ryan, R. M. (2000). The «what» and «why» of goal pursuits: human needs and the self-determination of behavior. Psychological Inquiry, 11, 227-268.

Deci, E. L., \& Ryan, R. M. (2008a). Self-determination theory: A machrotheory of human motivation, development, and health. Canadian Psychology, 49(3), 182-185.

Deci, E. L., \& Ryan, R. M. (2008b). Facilitating optimal motivation and psychological wellbeing across life's domains. Canadian Psychology, 49, 14-23.

Diener, E., \& Diener, M. (2009). Cross-cultural correlates in life satisfaction and self-esteem. En E. Diener (Ed.), Culture and well-being: The collected works of Ed Diener. Social Indicators Research Series 38, 71-91.

Diener, E., Emmons, R., Larsen, R. J., \& Griffin, S. (1985). The Satisfaction with Life Scale. Journal of Personalitiy Assessment, 49, 71-75.

Diener, E., Suh, E. M., Lucas, R. E., \& Smith, H. L. (1999). Subjective well-being: Three decades of progress. Psychological Bulletin, 125, 276-302.

Duda, J. L. (2005). Motivation in sport: The relevance of competence and achievement goals. In A. J. Elliot \& C. S. Dweck (Eds.), Handbook of competence and motivation (pp. 318-335). New York: Guilford Press.

Elliot, A. J. (1999). Approach and avoidance motivation and achievement goals. Educational Psychologist, 34, 169-189.

Elliot, A. J. (2005). A conceptual history of the achievement goal construct. In A. Elliot \& C. Dweck (Eds.), Handbook of competence and motivation (pp. 52-72). New York: Guilford Press.

Elliot, A. J., \& McGregor, H. A. (2001). A 2 x 2 achievement goal framework. Journal of Personality and Social Psychology, 80, 501-519.

Elmore, G. M., \& Huebner, E. S. (2010). Adolescents' satisfaction with school experiences: Relationships with demographics, attachment relationships, and school engagement behavior. Psychology in the Schools, 47, 525-537. 
Finney, S. J., \& DiStefano, C. (2006). Non-normal and categorical data in SEM. En G. R. Hancock \& R. O. Mueller (Eds.), Structural Equation Modelling: A second course (pp.269-314). Greenwich, CO: Information Age Publishing.

Fox, K. R. (1997). The physical self: from motivation to well-being. Champaign, IL: Human Kinetics.

Franco, E., Coterón, J., Gómez, V., Brito, J., \& Martínez, H. A. (2017). Influencia de la motivación y del flow disposicional sobre la intención de realizar actividad físico-deportiva en adolescentes de cuatro países. Retos, 31, 46-51.

Galand, B., Boudrenghien, G., \& Rose, A. (2012). Buts personnels, orientations motivationnelles et bien-être subjectif: Effets indépendants ou médiatisés? Canadian Journal of Behavioural Science, 44(2), 158167.

Gallagher, E. N., \& Vella-Brodrick, D. A. (2008). Social support and emotional intelligence as predictors of subjective well-being. Personality and Individual Differences, 44, 1551-1561.

Georgiadis, M. M., Biddle, S. J. H., \& Chatzisarantis, N. (2001). The mediating role of self-determination in the relationship between goa orientations and physical self-worth in Greek exercisers. European Journal of Sport Science, 1, 1-9.

González, J., Garcés de los Fayos, E., \& García, A. (2012). Indicadores de bienestar psicológico percibido en alumnos de Educación Física. Revista de Psicología del Deporte, 21(1), 183-187.

Granero-Gallegos, A., \& Baena-Extremera, A. (2014). Predicción de la motivación autodeterminada según las orientaciones de meta y el clima motivacional en EF. Retos, 25, 23-27.

Granero-Gallegos, A., Baena-Extremera, A., Pérez-Quero, F. J., OrtizCamacho, M. M., \& Bracho-Amador, C. (2014). Validación española del «intention to partake in leisure-time physical activity». Retos, 26, $40-54$

Guan, J., Xiang, P., McBride, R., \& Bruene, A. (2006). Achievement goals, social goals and students' reported persistence and effort in high school physical education. Journal of Teaching in Physical Education, 25, 58-74.

Gunnell, K. E., Crocker, P. R. E., Mack, D. E., Wilson, P. M., \& Zumbo, B. D. (2014). Goal contents, motivation, psychological need satisfaction, well-being and physical activity: A test of self-determination theory over 6 months. Psychology of Sport and Exercise, 15, 19-29.

Gutiérrez, M. (2014). Relaciones entre el clima motivacional, las experiencias en educación física y la motivación intrínseca de los alumnos. Retos, 26, 9-14.

Gutiérrez, M., \& Ruiz, L. M. (2009). Perceived motivational climate, sportsmanship, and students' attitudes toward physical education classes and teachers. Perceptual and Motor Skills, 108, 308-326.

Halvari, H., Skjesol, K., \& Bagøien, T E. (2011) Motivational climates, achievement goals, and physical education outcomes: A longitudina test of achievement goal theory. Scandinavian Journal of Educational Research, 55(1), 79-104.

Hu, L., \& Bentler, P. M. (1999). Cut-off criteria for fit indexes in covariance structure analysis: conventional criteria versus new alternatives. Structural Equation Modeling, 6, 1-55.

Isorna, M., Rial, A., \& Vaquero, R. (2014). Motivaciones para la práctica deportiva en escolares federados y no federados. Retos, 25, 80-84.

Jaakkola, T., Ntoumanis, N., \& Liukkonen, J. (2016). Motivational climate, goal orientation, perceived sport ability, and enjoyment within Finnish junior ice hockey players. Scandinavian Journal of Medicine \& Science in Sports, 26, 109-115.

Kaplan, D. (2000). Structural equation modeling: Foundations and extensions. Thousand Oaks, CA: Sage.

Long, B. C., \& van Stavel, R. (1999). Effects of exercise training on anxiety: a metaanalysis. Journal of Applied Sport Psychology, 7, 167189.

Lu, C., \& Buchanan, A. (2014). Developing students’ emotional wellbeing in physical education. Journal of Physical Education, Recreation \& Dance, 85(4), 28-33.

MacKinnon, D. P. (2008). Introduction to statistical mediation analysis. New York, NY: Routledge.

Moreno, J. A., González-Cutre, D., \& Sicilia, A. (2008). Metas de logro 2 x 2 en estudiantes españoles de Educación Física. Revista de Educación, 347, 299-317.

Nicholls, J. (1989). The competitive ethos and democratic education. Cambridge, MA: Harvard University Press.

Nunnally, J. C. (1978). Psychometric theory. New York: McGraw-Hill.

Núñez, J. L., León, J., González, V. \& Martín-Albo, J. (2011). Propuesta de un modelo explicativo del bienestar psicológico en el contexto deportivo. Revista de Psicología del Deporte, 20(1), 223-242.
Núñez, J. L., Martín-Albo, J., \& Domínguez, E. (2010). Propiedades psicométricas de la Escala de Satisfacción con la Vida en sujetos practicantes de actividad física. Revista de Psicología del Deporte, 19(2), 291-304.

Ommundsen, Y. (2006). Pupils' self-regulation in physical education: The role of motivational climates and differential achievement goals. European Physical Education Review, 12(3), 289-315.

Ortega, F. B., Artero, E. G., Ruiz, J. R., España, V., Jiménez, D., Vicente, G., ... \& HELENA group (2011). Physical fitness levels among European adolescents: the HELENA study. British Journal of Sports Medicine, 45(1), 20-29.

Reigal, R., Videra, A., \& Gil, J. (2014). Práctica física, autoeficacia general y satisfacción vital en la adolescencia. Revista Internacional de Medicina y Ciencias de la Actividad Física y el Deporte, 14(55), 561-576.

Rink, J. E. (2010). Teaching physical education (6th ed.). NY: McGraw Hill.

Roberts, G. C. (2001). Understanding the dynamics of motivation in physical activity: The influence of achievement goals on motivational processes. En G. C. Roberts (Ed.), Advances in motivation in sport and exercise (pp.1-50). Champaign, IL: Human Kinetics.

Ruiz-Juan, F., \& Baena-Extremera, A. (2015). Predicción de las metas de logro en educación física a partir de la satisfacción, la motivación y las creencias de éxito en el deporte. Revista Iberoamericana de Psicología del Ejercicio y el Deporte, 10(2), 193-203.

Ryan, R. M., \& Deci, E. L. (2000). Self-determination theory and the facilitation of intrinsic motivation, social development, and well-being. American Psychologist, 55, 68-78.

Ryan, R., \& Deci, E. (2001). On happiness and human potentials: A review of research on hedonic and eudaimonic well-being. Annual Review of Psychology, 52, 141-166.

Ryan, R. M., \& Deci, E. L. (2002). An overview of self-determination theory. In E. L. Deci, \& R. M. Ryan (Eds.), Handbook of selfdetermination research (pp. 3-33). Rochester, NY: University of Rochester Press.

Sistkos, B. N., Papaioannou, A. G., \& Propios, M. K. (2011). Causal effects between emotional intelligence and a perceived caring classroom climate in physical education. International Journal of Physical Education, 48, 7-17.

Smith, R E., Smoll, F. L., \& Cumming, S. P. (2009). Motivational climate and changes in young athletes' achievement goal orientations. Motivation \& Emotion, 33, 173-183.

Standage, M., Gillison, F. B., Ntoumanis, N., \& Treasure, D. C. (2012). Predicting students' physical activity and health-related well-being: A prospective cross-domain investigation of motivation across school physical education and exercise settings. Journal of Sport \& Exercise Psychology, 34, 37-60.

Standage, M., \& Gillison, F. (2007). Students' motivational responses toward school physical education and their relationship to general selfesteem and health-related quality of life. Psychology of Sport and Exercise, 8, 704-721.

Sun, R. C., \& Shek, D. T. (2012). Positive youth development, life satisfaction and problem behaviour among Chinese adolescents in Hong Kong: A replication. Social Indicators Research, 105, 541-559.

Taylor, I. M., Spray, C. M., \& Pearson, N. (2014). The influence of the physical education environment on children's well-being and physical activity across the transition from primary to secondary school. Journal of Sport \& Exercise Psychology, 36, 574-583.

Vansteenkiste, M., Niemiec, C. P., \& Soenens, B. (2010). The development of the five mini-theories of self-determination theory: an historical overview, emerging trends, and future directions. In T. Urdan, \& S. Karabenick (Eds.), The decade ahead: Advances in motivation and achievement (pp. 105-166). UK: Emerald Publishing.

Wilson, P. M., Rodgers, S., Fraser, W., Murray, T. C., \& McIntyre, C. A. (2004). The relationship between psychological need satisfaction and self-perceptions in females. Journal of Sport and Exercise Psychology, 26, S200.

Yeung, D. C., Yuan, X., Hui, S. S., \& Ferresu, S. A. (2016). Determinant of moderate to vigorous physical activity and obesity in children: a structural equation modeling analysis. World Journal of Pediatrics, 12(2), 170-176.

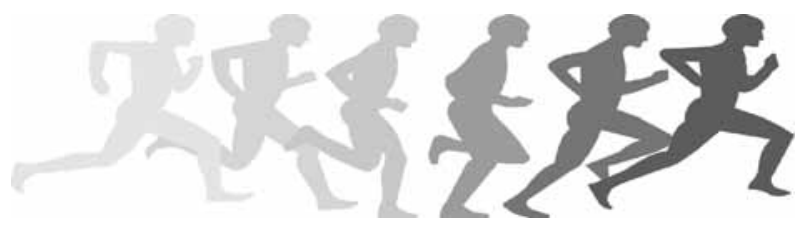

